\title{
Dynamics of solvent-free grafted nanoparticles
}

\author{
Alexandros Chremos, ${ }^{1}$ Athanassios Z. Panagiotopoulos, ${ }^{1, \text { a) }}$ and Donald L. Koch ${ }^{2}$ \\ ${ }^{1}$ Department of Chemical and Biological Engineering, Princeton University, \\ Princeton, New Jersey 08544, USA \\ ${ }^{2}$ School of Chemical and Biomolecular Engineering, Cornell University, Ithaca, New York 14853, USA
}

(Received 7 October 2011; accepted 4 January 2012; published online 26 January 2012)

\begin{abstract}
The diffusivity and structural relaxation characteristics of oligomer-grafted nanoparticles have been investigated with simulations of a previously proposed coarse-grained model at atmospheric pressure. Solvent-free, polymer-grafted nanoparticles as well as grafted nanoparticles in a melt were compared to a reference system of bare (ungrafted) particles in a melt. Whereas longer chains lead to a larger hydrodynamic radius and lower relative diffusivity for grafted particles in a melt, bulk solvent-free nanoparticles with longer chains have higher relative diffusivities than their short chain counterparts. Solvent-free nanoparticles with short chains undergo a glass transition as indicated by a vanishing diffusivity, diverging structural relaxation time and the formation of body-centered-cubic-like order. Nanoparticles with longer chains exhibit a more gradual increase in the structural relaxation time with decreasing temperature and concomitantly increasing particle volume fraction. The diffusivity of the long chain nanoparticles exhibits a minimum at an intermediate temperature and volume fraction where the polymer brushes of neighboring particles overlap, but must stretch to fill the interparticle space. (O) 2012 American Institute of Physics. [doi:10.1063/1.3679442]
\end{abstract}

\section{INTRODUCTION}

Nanoparticles are important both from a fundamental perspective and a practical point of view, being central to the development of novel photonic or structural materials. ${ }^{1-3}$ Small amounts of nanoscale inorganic additives in polymers can lead to disproportionate improvements in properties ${ }^{1}$ ranging from mechanical ${ }^{4}$ to fire resistance. ${ }^{5}$ Exotic behaviors such as defect "self-healing" are also possible. ${ }^{6}$ Despite significant progress in understanding nanocomposites, issues of poor miscibility and inadequate dispersion still prevent their widespread use. A common strategy to suppress aggregation is a functionalization by grafting polymer chains on the nanoparticles, typically using a polymer chemically identical to the matrix chains. ${ }^{7,8}$ Characteristics of the particles, such as the length and surface density of the grafted chains, not only influence the particle-polymer compatibility, but also affect the interparticle interactions and thereby the dynamical behavior of the whole system. ${ }^{8,9}$

An issue of fundamental importance, which has not been addressed previously in simulations, is the dynamical behavior of grafted nanoparticles in the absence of free chains or solvent. Experimental realization of such solvent-free composite systems has been recently demonstrated in the form of "nanoparticle organic hybrid materials" (NOHMs). ${ }^{10-15}$ These are core-shell systems consisting of a hard (inorganic) core and a soft (organic) oligomer corona. By varying the molecular weight and grafting density of the arms (corona), and the core particle size, they can display properties that span from glasses, stiff waxes, and gels (at high volume fractions), to simple liquids comprised of molecular building blocks (at

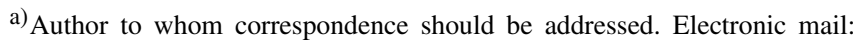
azp@princeton.edu.
}

low volume fractions). A highly attractive feature of these systems is the combination of inorganic and organic components to create hybrid particles as building blocks for the design of novel materials. The grafted chains prevent nanoparticle aggregation due to van der Waals attractions or depletion interactions, and also play the role of solvent. In recent studies, NOHMs structural features were obtained by field-theoretic methods ${ }^{16}$ and simulations. ${ }^{17}$ Recently, the structure and dynamics of solvent-free grafted nanoparticles subject to simple shear flows were examined in constant strain-rate and constant stress simulations. ${ }^{18}$ Solvent-free oligomer-grafted nanoparticles are significantly different from nanoparticles in a polymer matrix. In solvent-free conditions, the surrounding environment is composed of other grafted nanoparticles. For high enough grafting densities, the corona chains keep the nanoparticles well dispersed, and thus the nanoparticles can only interact with neighboring nanoparticles via their overlapping coronas. ${ }^{19}$ The parameters for the present study are the same as in previous work, ${ }^{17}$ in which the structure of bulk grafted nanoparticles was investigated. It was found that the corona chain length significantly influences the structure. For short chains, the systems displayed rich structural characteristics, while for longer chains the nanoparticles maintained a liquid-like structure over the whole temperature range explored. The primary aim of the present study is to investigate the dynamics of these systems.

Aside from our earlier work, ${ }^{16,17}$ previous theoretical and computational studies of oligomer-grafted nanoparticles mainly focused on self-assembly in solution, and often incorporated some degree of asymmetry in the shape of the core building blocks (e.g., spheres ${ }^{20,21}$ disks, ${ }^{22}$ cubes,${ }^{23}$ and $\operatorname{rods}^{24,25}$ ). Oligomer-grafted nanoparticles have been considered in previous theoretical studies in a phantom solvent to determine the effects of single ${ }^{26}$ and multiple grafted chains ${ }^{27}$ 
on their structure, and calculations of the particle-particle potential of mean force of grafted particles have been performed in an explicit polymer matrix. ${ }^{28}$ There have been several experimental demonstrations of controlled self-assembly using oligomer-grafted nanoparticles. ${ }^{22,29,30}$ Nevertheless, a full description of the dynamical behavior of particles with a core-shell architecture in bulk conditions is still lacking. In the present study, we report on the dynamical properties of bulk oligomer-grafted nanoparticles using molecular dynamics (MD) simulations at zero-pressure conditions. We focus on the impact of particle architecture on the properties. This is accomplished by keeping the grafting density and core size fixed, and varying the length of the end-grafted polymer chains. In addition to bulk solvent-free nanoparticle fluids, we also study the dynamical behavior of nanoparticles with or without grafted chains in a polymer matrix. Comparisons among these three cases highlight differences between NOHMs and conventional nanocomposite systems.

The paper is organized as follows. Section II contains details of the coarse-grained model, and the simulation methods. Results for the structure and dynamics of nanoparticles are presented in Sec. III. Section IV concludes the report.

\section{MODELS AND SIMULATION METHODS}

The grafted nanoparticle model is that of Ref. 17. Particles consist of a spherical core and $f$ attached oligomer chains. Each oligomer is composed of $M$ spherical beads (or monomers) connected to form a chain. The core and monomers have different radii, $R_{\mathrm{c}}$ and $R_{\mathrm{b}}$, respectively. We set the diameter of the monomers as our unit of length, $2 R_{\mathrm{b}}=\sigma$. The core radius is $R_{\mathrm{c}}=2.5 \sigma$. The oligomer grafting density is fixed as $\rho_{\mathrm{s}}=f /\left(4 \pi R_{\mathrm{c}}^{2}\right)=0.32 \sigma^{-2}$ unless stated otherwise. Interactions between all pairs of monomers are described by a cut-and-shifted Lennard-Jones potential with cutoff distance, $r_{\mathrm{c}}=2.5 \sigma$ and potential well depth $\varepsilon$. The first bead of each chain is rigidly attached to the core surface. The rest of the monomers along a chain are connected to their neighbors via a harmonic spring, $V_{\mathrm{H}}(r)=k\left(r-l_{0}\right)^{2}$, where $r$ is the beadbead separation, $l_{0}=\sigma$ is the natural length of the spring, and $k=10000 \varepsilon / \sigma^{2}$ is the spring constant.

The core-core and core-monomer interactions are modeled by the purely repulsive Weeks-Chandler-Andersen (WCA) potential, ${ }^{31}$ taking into account the difference in particle size, ${ }^{32}$

$V_{\mathrm{WCA}}(r)=\left\{\begin{array}{rl}4 \varepsilon\left[\left(\frac{\sigma}{r-\Delta_{\mathrm{ij}}}\right)^{12}-\left(\frac{\sigma}{r-\Delta_{\mathrm{ij}}}\right)^{6}+0.25\right] & r \leq r_{\mathrm{m}} \\ 0 & r>r_{\mathrm{m}},\end{array}\right.$

where $r_{\mathrm{m}}=2^{1 / 6}\left(R_{\mathrm{c}}+R_{\mathrm{c}}\right)$ and $\Delta_{\mathrm{cc}}=2\left(R_{\mathrm{c}}-R_{\mathrm{b}}\right)$ for core-core interaction, and $r_{\mathrm{m}}=2^{1 / 6}\left(R_{\mathrm{c}}+R_{\mathrm{b}}\right)$ and $\Delta_{\mathrm{cb}}=\left(R_{\mathrm{c}}\right.$ $-R_{\mathrm{b}}$ ) for core-monomer interaction. The energy and interaction range parameters were chosen to be the same for all three interactions, so that $\varepsilon_{\mathrm{cc}}=\varepsilon_{\mathrm{cb}}=\varepsilon$ and $\sigma_{\mathrm{cc}}=\sigma_{\mathrm{cb}}=\sigma$. The mass of a particle scales linearly with volume, so that the ratio of core to polymer bead mass is $R_{\mathrm{c}}^{3} / R_{\mathrm{b}}^{3}$. The concentration of the cores can be described using the volume fraction, $\phi_{\mathrm{c}}=N(4 \pi / 3) R_{\mathrm{c}}^{3} / L^{3}$.

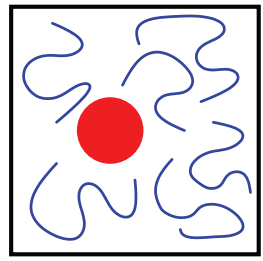

$\mathrm{N}$

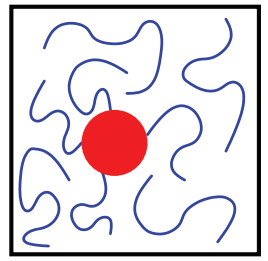

G

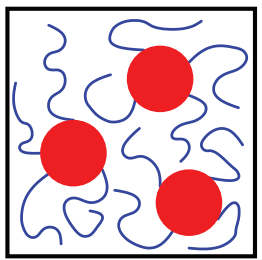

$\mathrm{S}$
FIG. 1. Schematic illustrations of the three classes of systems studied. From left to right we show an ungrafted particle in a polymer matrix, a grafted particle in a polymer matrix, and a neat (solvent-free) system consisting of many grafted particles. All simulations were for three-dimensional systems.

Simulations were performed in cubic boxes of length $L$; periodic boundary conditions and the minimum-image convention were applied in all three directions. The time step was set to $\delta t=0.004 \tau$, where $\tau$ is the unit of time, $\tau=\sigma \sqrt{m / \varepsilon}$, where $m$ is the mass of a polymer bead. The simulations were performed using the large-scale atomic/molecular massively parallel simulator, ${ }^{33}$ which takes advantage of a neighborlist construction and communication algorithm, speeding up simulations when the ratio of sizes of the particles becomes large..$^{34}$

The three classes of systems studied are shown schematically in Fig. 1.

The first class (denoted by "N") corresponds to a single nanoparticle without grafted oligomers in a polymer matrix of varying chain length. The second class ("G") consists of a grafted nanoparticle in a polymer matrix with chains of length equal to that of the grafted chains. The third type entails oligomer-grafted nanoparticles in the bulk ("S"). For all grafted particles we fix the number of attached chains, $f$, but vary their length, $M$, and temperature $T$. Temperature is measured in units of $\varepsilon / k_{\mathrm{B}}$, where $k_{\mathrm{B}}$ is Boltzmann's constant. We have studied chain lengths $M$ of 5,10, and 15 and temperatures from $T=1.0$ to 4.5 , always at a density corresponding to atmospheric pressure $(P=0)$ at the corresponding temperature.

To construct the system of interest we used the following process. For a nanoparticle in a polymer matrix we place randomly 1000 polymer chains and a nanoparticle in a simulation box with box size $L=200 \sigma$ such that there are no overlaps between the particles. Then we slowly compress the system $(0.01 \sigma$ per time step) until the desired density is reached. We then equilibrate the system for $2 \times 10^{6}$ time steps at a temperature such that the pressure is zero (atmospheric conditions). The production runs were $2 \times 10^{7}$ time steps. The process is the same for nanoparticles with and without grafted oligomers. For bulk conditions up to $N=450$ nanoparticles were used. The equilibration period for bulk systems was of $\mathrm{O}\left(10^{7} \delta t\right)$ and the production period of $\mathrm{O}\left(10^{7} \delta t\right)$.

The dynamical behavior was obtained by calculating the mean-square displacement of the core particles,

$$
\Delta r_{\mathrm{c}}^{2}(t)=\frac{1}{N}\left\langle\sum_{i=1}^{N}\left|\mathbf{r}^{i}(t)-\mathbf{r}^{i}(0)\right|^{2}\right\rangle,
$$

where the angled brackets denote an average over time origins. Once the mean-square displacement starts to exhibit a 
linear dependence on time, the diffusion coefficient can be obtained from the Einstein relation

$$
D_{\mathrm{i}}=\frac{\mathrm{d}\left(\Delta r_{\mathrm{c}}^{2}\right)}{6 \mathrm{~d} t}
$$

where the $D_{\mathrm{i}}$ is the diffusion coefficient with index i denoting the type of the system, $\mathrm{i}=\mathrm{N}, \mathrm{G}$, and $\mathrm{S}$.

\section{RESULTS AND DISCUSSION}

The simulations of an ungrafted nanoparticle in a melt involved a single particle diffusing in a unit cell with periodic boundary conditions. The Stokes-Einstein relationship

$$
D=\frac{T}{\eta \mu R_{\mathrm{c}}}
$$

implies that the diffusivity of a particle in a fluid matrix is related to the drag coefficient $\eta$ resisting the translational motion of the particle. Here, $\mu$ is the fluid viscosity and $\eta \mu R_{c}$ is the ratio of the drag force on a translating particle and its translational velocity $(\eta=6 \pi$ for a rigid particle in a continuum, unbounded fluid). Owing to the slow decay of the fluid velocity field due to a moving particle, Ladd showed that it was important to include the effects of periodic images on the drag when simulating particle diffusivity with periodic boundary conditions. ${ }^{35}$ The ratio of the diffusivity of a particle in a periodic cell to that in unbounded fluid is inversely proportional to the corresponding ratio of drag coefficients which has been calculated by Hasimoto ${ }^{36}$ and Sangani and Acrivos. ${ }^{37}$ When the volume fraction of the single core within the unit cell is small but finite, their results can be expressed as,

$$
\frac{D_{\text {Nbounded }}}{D_{\text {Nunbounded }}}=\frac{6 \pi}{\eta_{\text {bounded }}}=1-1.76 \phi_{\mathrm{c}}^{1 / 3}+\phi_{\mathrm{c}}-1.56 \phi_{\mathrm{c}}^{2} .
$$

Results for the diffusivity for a single nanoparticle without grafted chains in a polymer matrix are shown in Fig. 2. As one might expect, the diffusion coefficient of bare nanoparticles increases with increasing temperature and decreasing matrix chain length. The $D_{\mathrm{N}}$ and $D_{\mathrm{G}}$ were found to increase exponentially with $T$; we have fitted the simulations to exponential functions and when necessary use this fit to obtain the diffusion coefficient at the desired temperature. Small variations in simulated temperature obtained for different chain lengths are observed in Fig. 2. This occurred because the simulations for system types $\mathrm{N}$ and $\mathrm{G}$ with different chain lengths were performed at the same polymer bead number density rather than the same temperature.

We use the diffusivity $D_{\mathrm{N}}$ of an ungrafted nanoparticle in an unbounded melt as a reference value to which we compare the diffusivities of grafted particles. Thus, in Fig. 3, we plot the ratio $D_{\mathrm{G}} / D_{\mathrm{N}}$ and $D_{\mathrm{S}} / D_{\mathrm{N}}$, where $D_{\mathrm{G}}$ is the diffusivity of a grafted particle in a melt and $D_{\mathrm{S}}$ is the diffusivity of a grafted particle under bulk conditions. ${ }^{38}$ It can be seen that grafting chains to a particle leads to a moderate decrease in its diffusivity in a melt, but that the diffusivity of grafted particles under bulk conditions is reduced by one to two orders of magnitude.

It is known that the state of swelling of the brush on a particle is influenced by the ratio of the grafted chain length

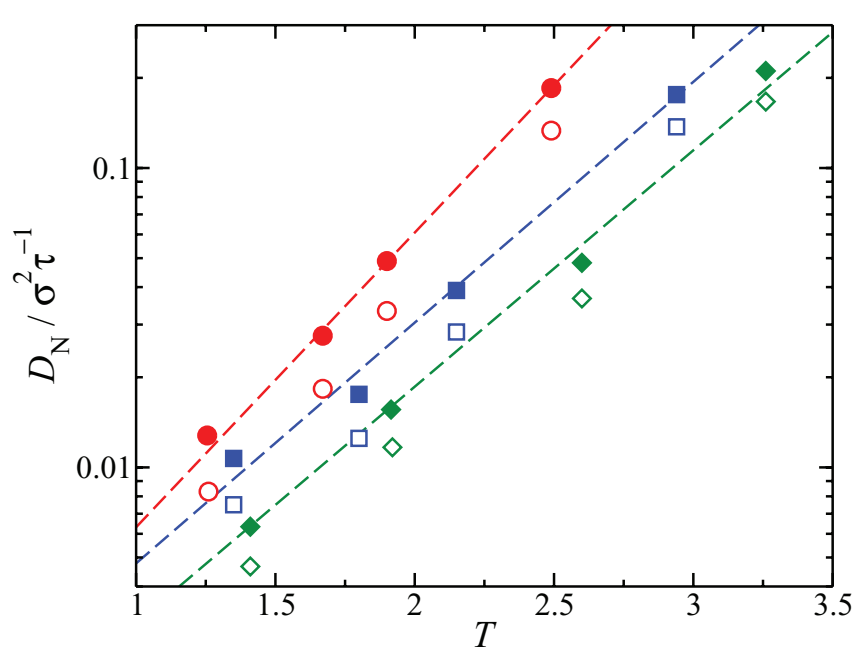

FIG. 2. Diffusion coefficients versus temperature for bare particles in a polymer matrix (system "N") with (solid symbols) and without (open symbols) corrections for periodicity according to Eq. (5). Circles are for chain length $M=5$, squares for $M=10$, and diamonds for $M=15$. The dashed lines are exponential fits of the form $D_{\mathrm{N}}=\alpha \exp (T / \beta)$, where $\alpha$ and $\beta$ are fitting parameters.

to the polymer matrix chain length with smaller matrix chains swelling the brush (wet brush) and larger matrix chains expelled from the brush (dry brush). ${ }^{8,39,40}$ However, we considered only the case of equal brush and matrix chain lengths which is at the transition between wet and dry brushes, since this case is most comparable to the bulk grafted nanoparticle case where the chains of one particle interact with chains of equal length tethered to neighboring particles.

A grafted nanoparticle in a melt, for the same temperature, has a lower diffusivity than a bare nanoparticle in the same polymer matrix. This is understandable, since the grafted chains on a nanoparticle increase its effective size and thus its hydrodynamic radius. To determine the total size of the particle, including the core and the polymer brush, we calculated the mean square end-to-center distance of grafted

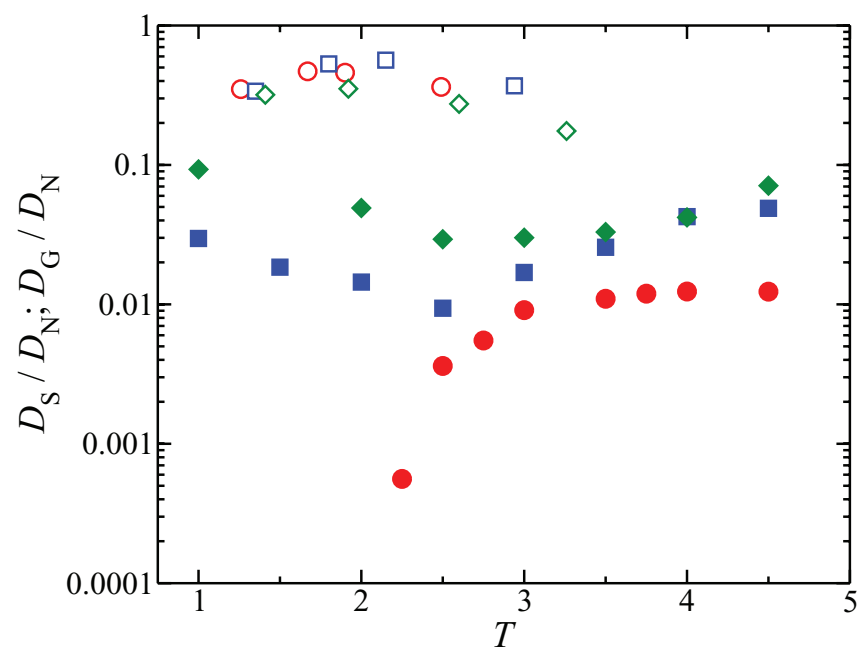

FIG. 3. Temperature dependence of the ratios of diffusivities of grafted particles in a polymer matrix, $D_{\mathrm{G}}$ (open symbols) and bulk grafted particles, $D_{\mathrm{S}}$ (solid symbols) to the diffusivity of bare particles, $D_{\mathrm{N}}$. Chain lengths are denoted using the same convention as for Fig. 2. 
nanoparticles $\left\langle R_{\mathrm{ec}}^{2}\right\rangle$ defined as

$$
\left\langle R_{\mathrm{ec}}^{2}\right\rangle=\frac{1}{f N}\left\langle\sum_{i=1}^{N} \sum_{j=1}^{f}\left|\mathbf{r}_{\mathrm{c}}^{i}-\mathbf{r}_{\mathrm{e}}^{i j}\right|^{2}\right\rangle,
$$

where $\mathbf{r}_{\mathrm{c}}^{i}$ is the position of core $i$ and $\mathbf{r}_{\mathrm{e}}^{i j}$ is the free end of chain $j$ of core $i$. The angle brackets correspond to an average over time.

It is common practice to use measurements of transport properties of particles in dilute suspensions to characterize the size of the particles. The diffusivity of the particle determined by dynamic light scattering or the intrinsic viscosity measured using capillary rheometry ${ }^{8,41,42}$ provides two common means of determining particle size. Using the measured diffusivity of a grafted particle in an unbounded polymer melt, we can define the effective hydrodynamic radius, as

$$
D_{\text {Gunbounded }}=\frac{T}{6 \pi \mu R_{\mathrm{h}}} .
$$

Accounting for periodicity effects using Eq. (5).

$$
\begin{gathered}
D_{\text {Gbounded }} / D_{\text {Nunbounded }}= \\
\left(\frac{R_{\mathrm{c}}}{R_{\mathrm{h}}}\right)\left(1-1.76 \phi_{\mathrm{h}}^{1 / 3}\right. \\
\left.+\phi_{\mathrm{h}}-1.56 \phi_{\mathrm{h}}^{2}\right), \\
\phi_{\mathrm{h}}=\frac{4}{3} \pi\left(R_{\mathrm{h}} / L\right)^{3} .
\end{gathered}
$$

Using the simulated value of $D_{\text {Gbounded }}$, Eqs. (8) and (9) can be solved to determine $R_{\mathrm{h}}$.

The low Reynolds number flow in the melt induced by a polymer-grafted particle would be expected to resist rapid variations in fluid velocity, so that the solvent within the polymer brush would move with the particle and its brush. As a result, one might expect the hydrodynamic radius of the particle to provide a good estimate of the size of the nanoparticle and its polymer brush. The results shown in Fig. 4 indicate that the hydrodynamic radius, $R_{\mathrm{h}}$, is indeed comparable with $\left\langle R_{\mathrm{ec}}^{2}\right\rangle^{1 / 2}$ and both increase with increasing length of the polymer brush. The root-mean-square end-to-center distance, $\left\langle R_{\mathrm{ec}}^{2}\right\rangle^{1 / 2}$, is nearly independent of temperature for $T$ $<2$ and grows with temperature for $T>2$. As the temperature increases, the void space between the polymer beads increases and this void space may be considered to be filled with a phantom solvent that swells the polymer brush. In Ref. 17 , we showed that one effect of this phantom solvent was to swell the polymer brushes in a bulk nanoparticle system and decrease the tendency of neighboring brushes to overlap one another. The hydrodynamic radius, $R_{\mathrm{h}}$, does not increase with increasing $T$ as much as $\left\langle R_{\mathrm{ec}}^{2}\right\rangle^{1 / 2}$. It is possible that the phantom solvent that swells the polymer brushes expels the melt polymers and induces a slip between the particle and the polymer melt that increases the diffusivity and decreases $R_{\mathrm{h}} /\left\langle R_{\mathrm{ec}}^{2}\right\rangle^{1 / 2}$.

In bulk conditions, the grafted nanoparticles interact with each other in the absence of free chains. Figure 3 shows the ratio of the diffusivity of bulk grafted nanoparticles to that of bare nanoparticles in a polymer chain melt, $D_{\mathrm{S}} / D_{\mathrm{N}}$. Grafted nanoparticles in bulk conditions have significantly smaller

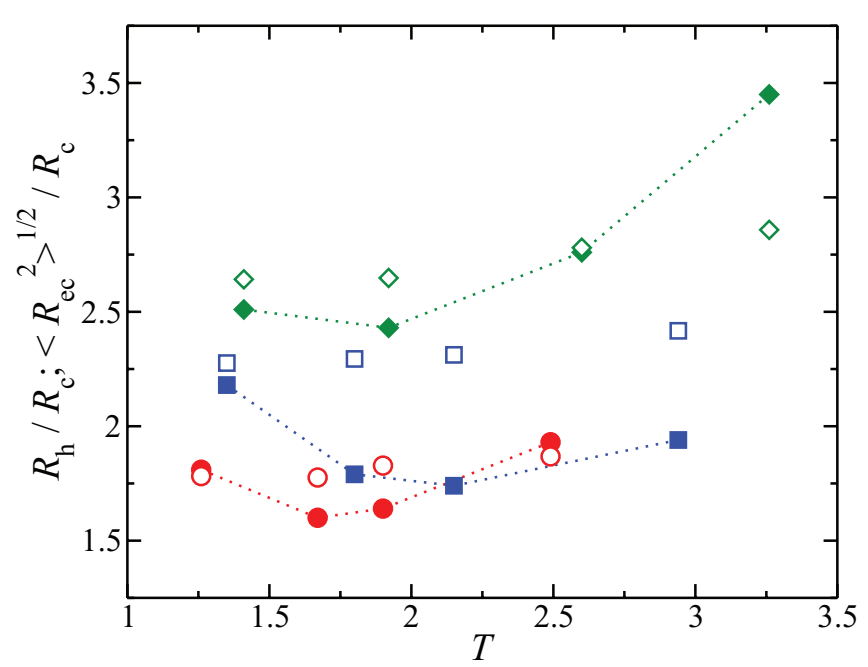

FIG. 4. The hydrodynamic radius, $R_{\mathrm{h}}$, of a single grafted nanoparticle in a polymer matrix (open symbols) and the root-mean-square end-to-center distance, $\left\langle R_{\mathrm{ec}}^{2}\right\rangle^{1 / 2}$ (solid symbols and dotted lines). Circles are for chain length $M=5$, squares for $M=10$, and diamonds for $M=15$.

diffusion coefficients than in a polymer matrix, but are still diffusive liquids despite the absence of solvent. The diffusivity ratio, $D_{\mathrm{S}} / D_{\mathrm{N}}$, is larger for longer grafted polymer chains. This surprising trend can be understood qualitatively in terms of the density-functional theory of Ref. 17. In that theory, the grafted polymer chains are required to fill the interstitial space between the cores. Short stiff polymers experience a higher entropic penalty when satisfying this constraint than longer polymers with the result that shorter polymers lead to stronger polymer-induced particle-particle interactions. Longer polymers which can more easily fill the interstitial space lead to a more fluid environment.

The temperature dependence of the dynamical behavior in bulk conditions differs significantly from that in a polymer matrix. For $M=5$ at high values of temperature, there is little variation in diffusion coefficient ratio, but for $T<3$, the ratio decreases rapidly with decreasing temperature. The lowest temperature computationally accessible for this system was $T=2.25$ at which solid-like characteristics were manifested with a highly pronounced first peak and a split in the second peak, of the pair correlation function, as shown in Fig. 5. A detailed discussion of the structural behavior of bulk grafted nanoparticles can be found in Ref. 17. We include in Fig. 5 peaks for a perfect body-centered cubic (BCC) crystal structure, scaled so that the first peak coincides with the first peak of the radial distribution function of the grafted nanoparticles. The agreement in location of higher order peaks suggests that the nanoparticles organized in a body-centered-cubic structure.

For the two longer chain lengths of $M=10$ and 15, the ratio $D_{\mathrm{S}} / D_{\mathrm{N}}$ exhibits a minimum at a certain temperature, $T_{\min }$ $\approx 2.5$. In our previous work, ${ }^{17}$ we found that these nanoparticle systems underwent important structural transitions at the same temperature. The height of the first peak of the pair distribution function was larger at this temperature than at either higher or lower temperatures, suggesting that particle interactions mediated by the polymer brush or corona are 


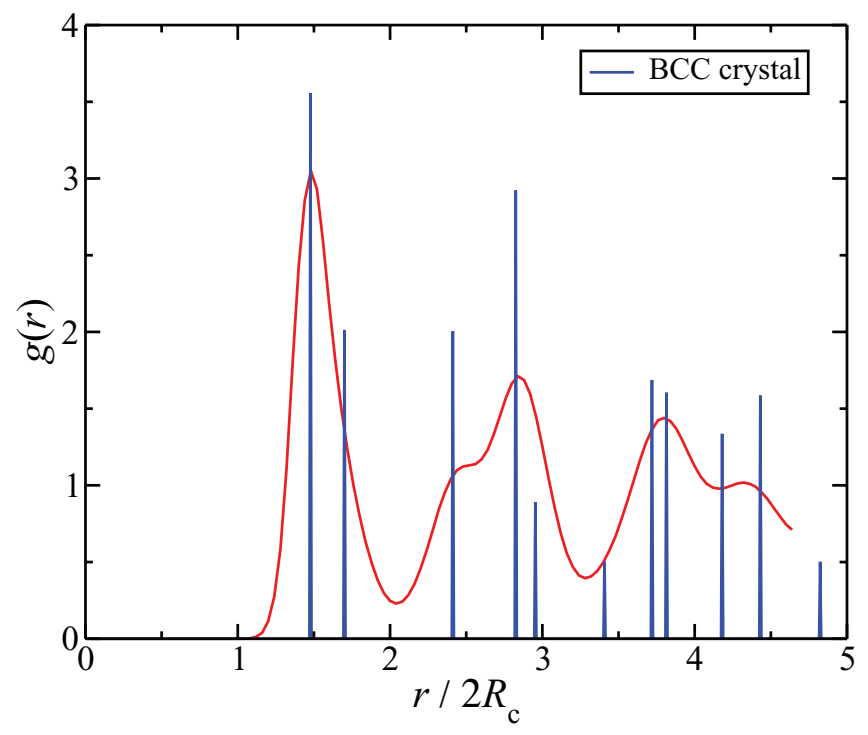

FIG. 5. Radial distribution function for bulk grafted nanoparticles with $M=$ 5 at $T=2.25$, at which a BCC crystal structure is formed. The nanoparticle volume fraction was $\phi_{\mathrm{c}}=0.2$.

particularly strong at this intermediate temperature. As the temperature increases, the system expands and the volume fraction of the core particles decreases, as shown in Fig. 6(a). The corona surrounding the particles also expands in the bulk system. However, the interpenetration between the brushes of neighboring nanoparticles remains nearly constant for $T<$ 2.5 and decreases with increasing temperature for $T>2.5 .^{17}$ When the temperature increases from $T=1$ to 2.5 , the volume fraction decreases and the chains on neighboring particles expand in a cooperative fashion to fill the interparticle space. The entropic penalty for filling an increased interstitial space is larger so that the particle interactions are stronger at lower volume fractions. ${ }^{16}$ At temperatures larger than $T_{\min }$, the polymer brushes can be thought of as being swollen by the phantom solvent so that they tend to expel the polymer brushes from the neighboring particles. This may decrease

(a)

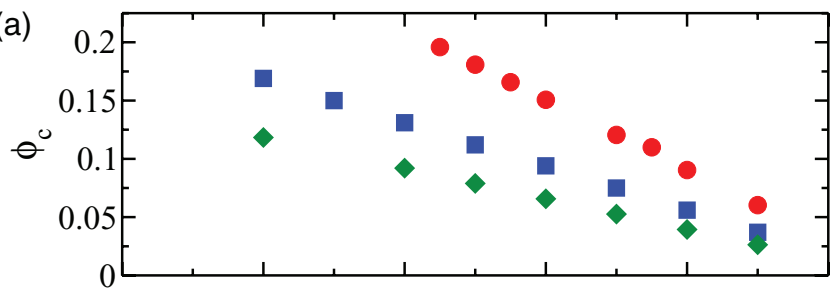

(b)

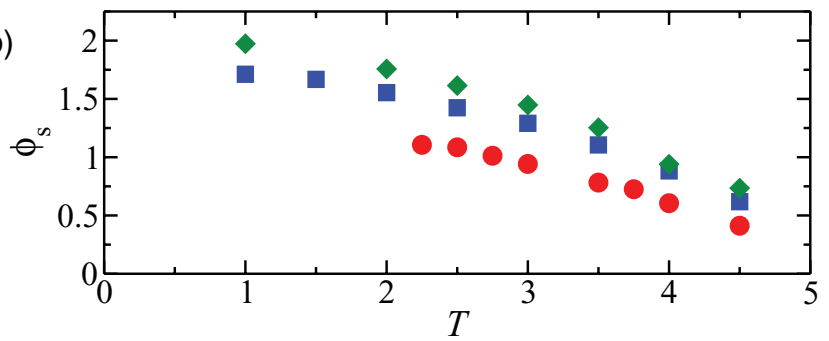

FIG. 6. (a) Nanoparticle volume fraction, $\phi_{\mathrm{c}}$ versus temperature. (b) Volume fraction of the core and corona, $\phi_{\mathrm{s}}$ versus temperature. Symbols are the same as in Fig. 2.

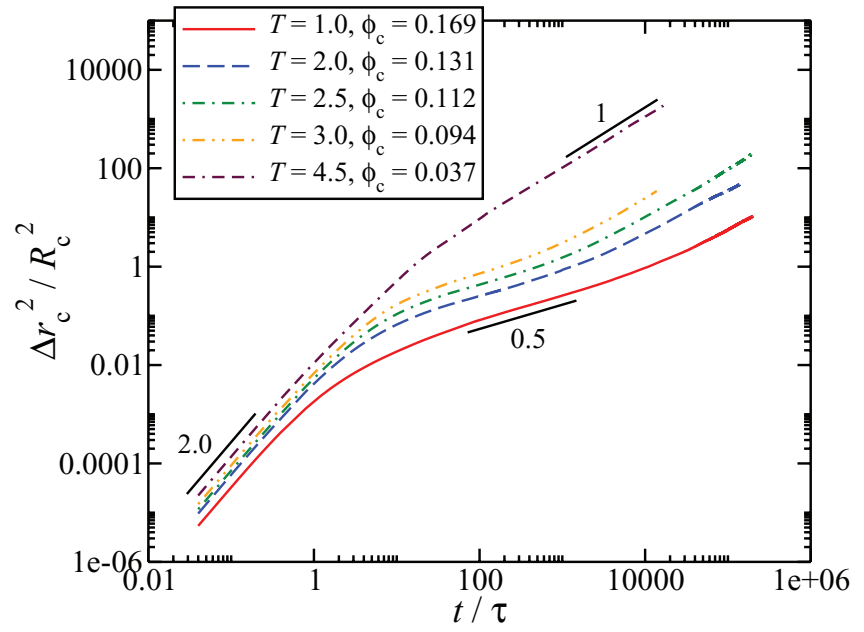

FIG. 7. Mean-square displacement of bulk grafted nanoparticles for $M=10$ at different temperatures.

the coupling between the motion of neighboring particles in a manner consistent with the decreased $R_{\mathrm{h}} /\left\langle R_{\mathrm{ec}}^{2}\right\rangle^{1 / 2}$ for grafted nanoparticles in a melt evident in Fig. 4.

The mean-square displacement (MSD) only achieves diffusive behavior after particles have moved a distance comparable to their core diameter. At low temperatures, this process requires an extended period of time, as illustrated in Fig. 7 where the mean-square displacement of the particles is plotted as a function of time for $M=10$ at different temperatures $T$. At short times, the particle motion is ballistic. At low temperatures, this regime is followed by an extended period of sub-diffusive behavior where the MSD grows as $t^{1 / 2}$. Finally at long times, the diffusive behavior, MSD $\sim t$ is observed. The density-functional theory of Ref. 17 showed that space filling polymer brushes can lead to a more attractive potential of mean force between core particles. This attraction may account in part for the sub-diffusive behavior. The frictional interaction of the interpenetrating polymer brushes may also play a role. At high temperatures, the interpenetration of the polymer brushes is reduced and the swollen polymer brush induced particle interactions are repulsive as indicated by the outward shift of the first peak of the pair probability in Fig. (6) of Ref. 17. The reduced interaction among the brushes may account for the simple transition of the MSD transitions from ballistic to diffusive regime with no intermediate subdiffusive regime.

The dynamical behavior of a particle suspension such as the bulk nanoparticle system can be described by the Van Hove self-correlation function, which is an experimentally observable quantity that measures the decay of density fluctuations. ${ }^{43}$ The function is written as

$$
F(q, t)=\frac{1}{N} \sum_{j=0}^{N}\left\langle\exp \left\{i \mathbf{q} \cdot\left[\mathbf{r}^{j}\left(t+t^{\prime}\right)-\mathbf{r}^{j}\left(t^{\prime}\right)\right]\right\}\right\rangle .
$$

The wave number $q$ in $F(q, t)$ can take on a range of values to reflect the decay of different Fourier modes of the particle density correlations. However, following a common choice, we consider the wavenumber corresponding to the first maximum in the static structure factor $S(q)$. Consistent 

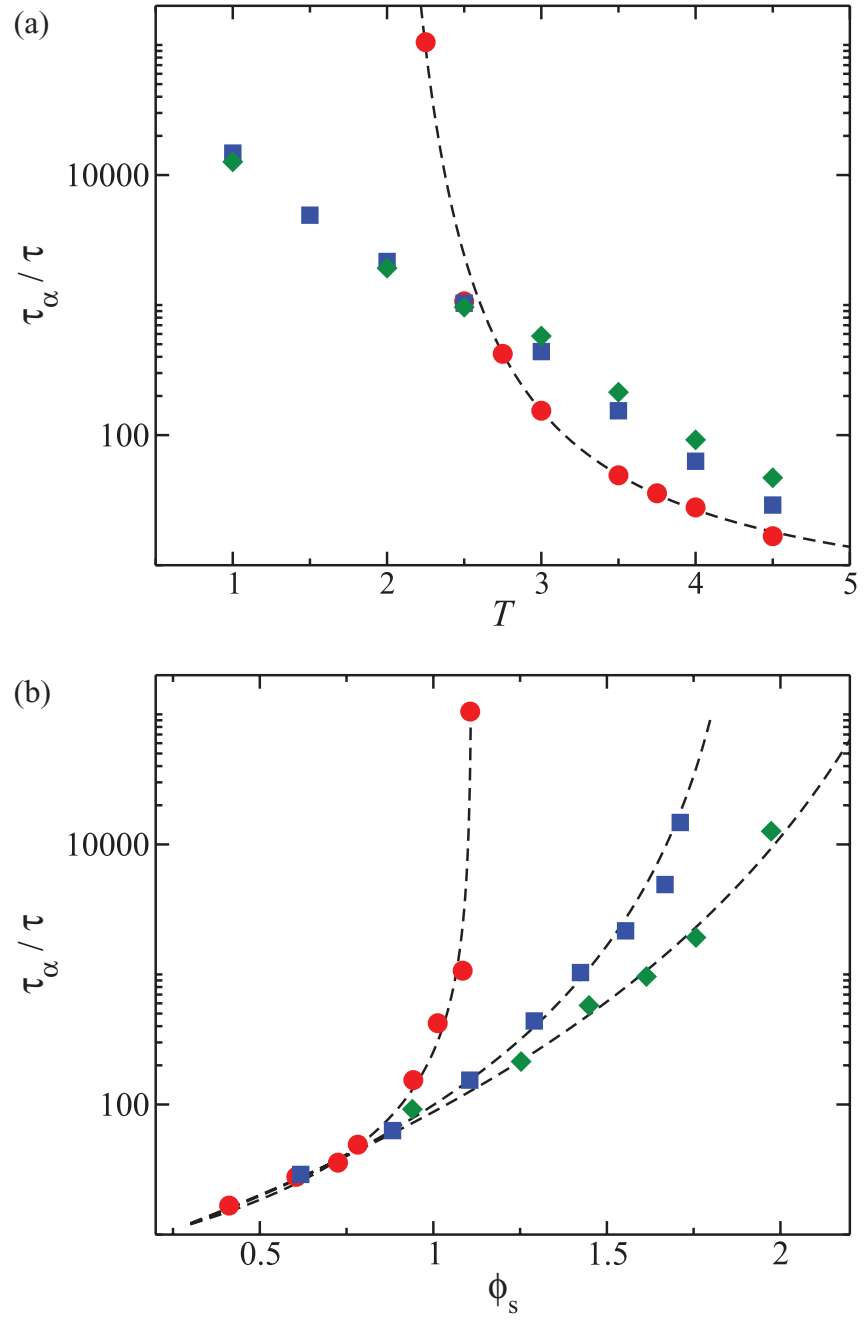

FIG. 8. Structural relaxation time of bulk oligomer-grafted nanoparticles for different chain lengths versus (a) temperature; (b) volume fraction of the core and corona, $\phi_{\mathrm{s}}$. The dashed line in (a) is a fit of the VFT relation as described by Eq. (11). The dashed lines in (b) are fitting lines based on Eq. (14) and their values are listed in Table I. Symbols are the same as in Fig. 2.

with the complex temporal behavior of the mean-squared displacement, we found that $F(q, t)$ does not follow a simple exponential decay but can be fit by stretched exponential functions as has been seen in molecular dynamics studies of other complex fluids near their glass transition. ${ }^{44}$ To provide a simpler measure of the decay of density correlation we define a structural relaxation time, $\tau_{\alpha}$, as the time at which the correlations have decreased by a factor of $e$, i.e., $F\left(q, \tau_{\alpha}\right)$ $=1 / e$. Results for $\tau_{\alpha}$ plotted in Fig. 8 reveal an interesting distinction between the behavior of particles with short corona $M=5$ and longer corona $M=10$ and 15 . The structural relaxation time for all chain lengths increases with decreasing temperature, but whereas the relaxation time diverges for $M$ $=5$ at a temperature near $T=2$, the increase in the relaxation time is much more gradual for $M=10$ and 15 . The structural relaxation time for fluids that undergo a liquid-solid transition is usually fit by the well-known Vogel-Fulcher-Tammann (VFT) relation

$$
\tau_{\alpha}=\tau_{\mathrm{v}} \exp \left(\frac{C}{T-T_{0}}\right)
$$

where $C$ and $T_{0}$ are parameters with dimensions of temperature, and $\tau_{\mathrm{v}}$ is the characteristic relaxation time for high values of temperature. $T_{0}$ is known as the Vogel temperature and is typically $30-50 \mathrm{~K}$ below $T_{\mathrm{g}}$, the glass transition temperature. For nanoparticles with $M=5$ the behavior of the structural relaxation time follows the VFT relation with $T_{0}$ $=1.8, C=4.626$, and $\tau_{\mathrm{v}}=3.226$, Fig. 8(a). This indicates that for $M=5$ the nanoparticles have a glass transition temperature in the vicinity of $T=2$. This happens because the length of the chain is rather short leaving the core to dominate the overall behavior and so the nanoparticles have a behavior more closely related to hard spheres or colloidal suspensions. Such systems form more compact structures and the particles rattle in traps formed by their neighbors in accordance with classical liquid dynamics. Moreover, hard-spherelike systems are also referred to as "fragile" because they exhibit rapid changes of the viscosity and $\alpha$-relaxation time close to glass transition temperature due to weak inter-particle forces. ${ }^{45}$ Soft particles are less sensitive to temperature variation as observed in recent experiments on soft colloids ${ }^{46}$ and are referred to as strong glasses/liquids. From Fig. 8(a) the structural relaxation time for $M=5$ is sensitive to temperature variation (diverging near $T=2$ ), while for longer chains $\tau_{\alpha}$ gradually increases and the grafted nanoparticles are less sensitive to temperature variation. A softer particle would not feel frustrated by the decrease of the interparticle space (by decreasing the temperature), since the soft overlapping coronas provide enough scope for interparticle motion to prevent caging by the neighboring nanoparticles and thus allow the nanoparticles to remain in the disordered phase and avoid crystallization. In particular, no evidence of a glass transition or crystallization was observed. This indicates the significant effects of the corona can have in bulk conditions.

The dynamical behavior of colloidal suspensions is usually studied as a function of particle concentration and for hard-sphere colloids the particle volume fraction is the only parameter controlling the dynamics. In NOHMs the volume fraction of the nanoparticle cores is much smaller than the volume fraction at which hard spheres start to crystallize, as discussed above. This suggests that the corona significantly contributes to the effective size of the particle. In particular, the current understanding is that soft particles with a small corona undergo the liquid-solid transition at a critical value of an effective volume fractions based on the combined size of the core and the corona. ${ }^{47}$ We define the effective volume fraction of the core and corona as

$$
\phi_{\mathrm{s}}=\phi_{\mathrm{c}}\left(\frac{\left\langle R_{\mathrm{ec}}^{2}\right\rangle^{1 / 2}}{R_{\mathrm{c}}}\right)^{3} .
$$

This approach has been discussed in connection with the behavior of grafted nanoparticles in a polymer matrix in Ref. 8 . The values of $\phi_{\mathrm{s}}$ may exceed unity due to the interpenetration of the coronas. Figure 8(b) shows how the structural relaxation time grows with increasing $\phi_{\mathrm{s}}$. Longer grafted chains display a more gradual increase. This is in good qualitative agreement with the observations for viscosity measurements of grafted nanoparticles in a polymer matrix when the effective volume fraction is varied by adding solvent, see Fig. 7(b) of Ref. 8. The structural relaxation time gives insights into 
TABLE I. Fitting parameters in Eq. (14) for the structural relaxation time versus $\phi_{\mathrm{s}}$.

\begin{tabular}{lcc}
\hline \hline$M$ & $\tau_{0}$ & $\phi_{\mathrm{sm}}$ \\
\hline 5 & 8.82 & 1.11 \\
10 & 6.40 & 1.96 \\
15 & 6.21 & 2.80 \\
\hline \hline
\end{tabular}

the viscosity, since viscosity can be thought of as resulting from temporal correlations of momentum fluctuations. The semi-empirical Krieger-Dougherty relation describes the relative viscosity, $\eta_{\mathrm{r}}$, as a function of the volume fraction, and has been successfully used to understand the viscosity of hard spheres,

$$
\eta_{\mathrm{r}}=\left(1-\phi_{\mathrm{c}} / \phi_{\mathrm{m}}\right)^{-[\eta] \phi_{\mathrm{m}}},
$$

where $\phi_{\mathrm{m}}$ is the limiting packing fraction and $[\eta]$ is the intrinsic viscosity. ${ }^{48}$ The Krieger-Dougherty relation has successfully predicted the viscosity of soft grafted nanoparticles in a melt by substituting the effective volume fraction $\phi_{s}$ in place of the core volume fraction $\phi_{c}$ in Eq. (13). ${ }^{49}$ With this in mind, we fit the structural relaxation results in Fig. 8(b) to an equation analogous to Eq. (13),

$$
\tau_{\alpha}=\tau_{0}\left(1-\phi_{\mathrm{s}} / \phi_{\mathrm{sm}}\right)^{-2.73 \phi_{\mathrm{sm}}+1.62},
$$

where $\tau_{0}, \phi_{\text {sm }}$ are fitting parameters. We find Eq. (14) to fit well with our results, see Fig. 8(b). The values of the fitting parameters are listed in Table I. The parameter $\phi_{\text {sm }}$ represents the volume fraction of the combined core and corona at which the relaxation time diverges. This may be expected to provide a rough estimate of the concentration at which the viscosity diverges. The values of $\phi_{\mathrm{sm}}$ increase as the grafted chain length increases. For grafted nanoparticles in a melt, the effective volume fraction is changed by adding or removing solvent. In the bulk nanoparticle system studied here, however, $\phi_{\mathrm{s}}$ varies due to changes in temperature and chain length as illustrated in Fig. 6(b). The effective volume fraction varies approximately linearly with temperature at low $T$ and it can be seen from Fig. 6(b) that the effective volume fraction $\phi_{\mathrm{s}}$ $=2.80$ at which the structural relaxation time would diverge for $M=15$ cannot be achieved even as $T \rightarrow 0$. This indicates that even at low temperatures, the interpenetrating coronas are able to maintain a liquid state in which the cores relax to a disordered equilibrium configuration. We might then expect this system to become glassy only near the glass transition temperature of non-entangled polymer chains, at about $T=0.4$. A similar picture applies for $M=10$ although the effective volume fraction achieved as $T \rightarrow 0$ is only slightly smaller than $\phi_{\text {sm }}$.

The discussion above highlights the features that govern the behavior of grafted nanoparticles and other core-shell architecture systems, namely a hard core and a fluid corona. It is possible that a portion of the corona may contribute to an enhanced effective hard core size. Reference 8 considers the viscosity of grafted nanoparticles in solvents and notes that nanoparticles whose corona size is small enough (about 0.3 times of the core radius) exhibit a viscosity that diverges at effective volume fractions comparable to that for hard spheres. For the bulk nanoparticle systems the ratio of the corona size to the radius of the core takes the values (averaged over the different temperatures explored) $0.85,1.37$, and 1.8 for $M=5,10$, and 15, respectively. For the shortest chains the bulk system resembles the behavior for hard spheres but the divergence occurs at a value of $\phi_{\mathrm{s}} \approx 1.1$ that is higher than that for hard spheres (0.64). Polymer brushes in a good solvent create a purely repulsive interparticle potential so that the brushes of neighboring particles tend not to overlap. ${ }^{47}$ However, corona-induced interparticle potentials in bulk nanoparticle systems exhibit both attractive and repulsive components so that the corona of neighboring particles overlap substantially especially at low temperatures. ${ }^{17}$ Thus, a portion of the corona contributes to the effective hard-sphere size while another portion acts as a fluid layer that suspends the nanoparticles.

In Fig. 3, we plotted the diffusivity in a solvent-free NOHMs system as a function of temperature. We have subsequently noted that the particle concentration varies with temperature and that the variation of the structural correlation time can be understood in terms of its dependence on an effective particle concentration. It is interesting then to consider the dependence of the diffusivity on particle concentration. Figure 9 shows the ratio of the diffusivity of solvent-free grafted nanoparticles to the diffusivity of an ungrafted particle in a melt as a function of the core volume fraction. It can be seen that the diffusivity for $M=5$ decreases monotonically with volume fraction and approaches zero at $\phi_{c}=0.21$. The diffusivity ratios for $M=10$ and 15 are larger and have a non-monotonic dependence on particle volume fraction. The dependence of the diffusivity of hard-sphere systems or systems with repulsive interactions on particle volume fraction is often fit with the Doolittle equation which is based on free volume theory, ${ }^{50,51}$

$$
\frac{D_{\mathrm{S}}}{D_{\mathrm{N}}}=\zeta \exp \left(-\frac{\kappa}{\left(\phi_{\mathrm{c}}\right)^{-1}-\phi_{\mathrm{cp}}^{-1}}\right),
$$

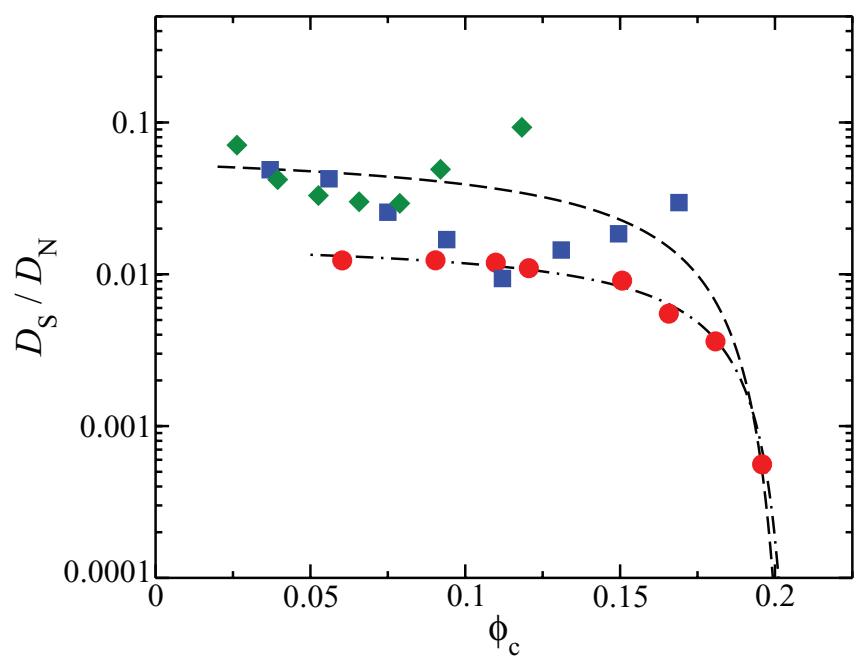

FIG. 9. Ratio of diffusion coefficient of $D_{\mathrm{S}}$ over $D_{\mathrm{N}}$ versus nanoparticle volume fraction, $\phi_{\mathrm{c}}$. The lines are fits of the data to Eq. (15) with $\phi_{\mathrm{cp}}=0.21$. The dashed line has $\kappa=1.041$ and $\zeta=0.0144$, and the dotted-dashed line has $\kappa=1.6$ and $\zeta=0.054$. Symbols are the same as in Fig. 2 . 
where $\kappa$ is a parameter and $\phi_{\mathrm{cp}}$ is the nanoparticle volume fraction at which the diffusivity goes to zero. We have included a pre-factor $\zeta$ to account for the fact that $D_{S}$ differs from $D_{N}$ even at low particle concentrations due to the frictional and potential interactions of overlapping polymer brushes. For hard spheres $\kappa=1.6, \phi_{\mathrm{cp}}=0.65$, and $\zeta=1$. For $M=5$ the Doolittle equation provides a good fit with $\kappa=$ $1.041, \phi_{\mathrm{cp}}=0.21$, and $\zeta=0.0144$. This suggests an effective hard-sphere radius that is larger than the core radius, $R_{\mathrm{eff}} / R_{\mathrm{c}}$ $=1.45$, and smaller than the root-mean-square end-to-center distance, e.g., $R_{\text {eff }} /\left\langle R_{\mathrm{ec}}^{2}\right\rangle^{1 / 2}=0.786$ for $T=3.0$. The behavior of the diffusivity for nanoparticles with longer chains $M$ $=10$ and 15 is poorly described by the Doolittle equation even if we increase $\zeta$ to 0.054 to account for the larger ratio $D_{S} / D_{N}$ exhibited by the long chain particles at low volume fraction. As noted above, the minimum in the diffusivity at an intermediate volume fraction which corresponds to an intermediate temperature arises because the particle interaction is strongest at intermediate temperatures. The subtle changes in the interactions of interpenetrating polymer brushes cannot be modeled by an effective hard-sphere system.

Star polymers are the limiting case of grafted nanoparticles when $R_{\mathrm{c}} /\left\langle R_{\mathrm{ec}}^{2}\right\rangle^{1 / 2} \ll 1$ and can display polymer- and colloidal-like behavior by tuning the number and length of the grafted chains. ${ }^{52-54}$ To our knowledge pure polymer star melt systems, the equivalent of our solvent-free grafted nanoparticles as $R_{\mathrm{c}} \rightarrow 0$, have not been studied. However, there are many experimental and theoretical studies of star polymers in both small molecule solvents and linear polymer melts. ${ }^{53,55-57}$ The softness of star polymers in solution affects their dynamical behavior as seen in measurements of relative viscosity versus the effective volume fraction in solution as seen, for example, in Fig. (6) of Ref. 57 (compare with Fig. 8(b)). Star polymers with a large number of arms exhibit that diverges at a critical concentration in a manner similar to hard-sphere colloids, but softer star polymers (smaller number of arms) display a slower viscosity increase. In our study of NOHMs we have tuned particle softness by varying $\left\langle R_{\mathrm{ec}}^{2}\right\rangle^{1 / 2} / R_{\mathrm{c}}$ and seen a similar transition from hard-sphere-like divergence of the structural relaxation time at small $\left\langle R_{\mathrm{ec}}^{2}\right\rangle^{1 / 2} / R_{\mathrm{c}}$ to a more gradual increase of relaxation time with concentration for longer chained NOHMs. The dynamical behavior of star polymers is currently understood in terms of the Milner-McLeish theory, which models the release of constraints on a test grafted chain, as other grafted chains reptate or retract away from its environment. ${ }^{58}$ Such an approach may potentially describe the grafted nanoparticles when chain length becomes very large.

\section{CONCLUSIONS}

In this work, computer simulations of bead-spring models have been used to gain insights into the dynamical behavior of polymer-grafted nanoparticles. The study was motivated by the unique physical features of NOHMs systems.

The transport properties of bulk oligomer-grafted nanoparticles were studied and valuable insights obtained by comparisons of the behavior of nanoparticles with and without grafted chains in a polymer matrix. In particular, grafted nanoparticles in bulk conditions have lower diffusivities than in a polymer matrix. In a polymer matrix, grafted nanoparticles have higher diffusivities relative to their bare nanoparticle counterparts when tethered oligomers are shorter. The opposite trend is observed for grafted-nanoparticles in bulk conditions where the diffusivity relative to a bare particle is larger for nanoparticles with longer oligomers. The diffusivity and structural relaxation time for solvent-free nanoparticles with shorter chains resemble those of hard spheres but with an enhanced effective particle size that includes a portion of the polymer brush. These systems experience an order-disorder transition even if the nanoparticles are too small to create ordered structures on their own. Longer grafted chains create a larger and softer corona, which filters the caging effects from neighboring nanoparticles so that no order forms within the parameter space explored. For soft grafted nanoparticles, the corona dominates the overall behavior. The diffusivity of these particles is higher at both low temperatures, where the chains can easily fill the intercore space, and high temperatures, where the polymer brushes of neighboring particles have little overlap than at an intermediate temperature, where the brushes overlap but the chains must stretch to fill the interparticle space. Overall, the chain length of the grafted chains on the nanoparticles tunes the size and the softness of the corona. For small and hard corona the particles behave similar to hard spheres, while for large and soft corona the nanoparticle character disappears in favor of a polymer melt of hyperbranched chains, with the nanoparticles acting as branching points.

\section{ACKNOWLEDGMENTS}

The authors would like to thank Professor Fernando Escobedo and Hsiu-Yu Yu for helpful discussions and Professor Escobedo for suggesting the simulation model used in this work. This publication is based on work supported in part by Award No. KUS-C1-018-02, made by King Abdullah University of Science and Technology (KAUST). Additional support was provided by grant CBET-1033155 from the U.S. National Science Foundation (NSF).

${ }^{1}$ Polymer Nanocomposites, edited by R. A. Vaia and E. P. Giannelis (American Chemical Society, Washington, 2001).

${ }^{2}$ E. V. Shevchenko, D. V. Talapin, N. A. Kotov, S. O'Brien, and C. B. Murray, Nature (London) 439, 55 (2006).

${ }^{3}$ M. Grzelczak, J. Vermant, E. M. Furst, and L. M. Liz-Marzán, ACS Nano 4, 3591 (2010)

${ }^{4}$ E. P. Giannelis, Adv. Mater. 8, 29 (1996).

${ }^{5}$ T. Kashiwagi, E. Grulke, J. Hilding, R. Harris, W. Awad, and J. Douglas, Macromol. Rapid Commun. 23, 761 (2002).

${ }^{6}$ S. Gupta, Q. Zhang, T. Emrick, A. Balazs, and T. P. Russel, Nature (London) 5, 229 (2006).

${ }^{7}$ G. Lindenblatt, W. Gchärtl, and M. Schmidt, Macromolecules 33, 9340 (2000).

${ }^{8}$ M. McEwan and D. Green, Soft Matter 5, 1705 (2009).

${ }^{9}$ L. Leibler and P. A. Pincus, Macromolecules 17, 2922 (1984).

${ }^{10}$ J. L. Nugent, S. S. Moganty, and L. A. Archer, Adv. Mater. 22, 3677 (2010).

${ }^{11}$ A. B. Bourlinos, R. Herrera, N. Chalkias, D. D. Jiang, L. A. Archer, and E. P. Giannelis, Adv. Mater. 17, 234 (2005).

${ }^{12}$ A. B. Bourlinos, S. R. Chowdhury, R. Herrera, N. Chalkias, D. D. Jiang, L. A. Archer, and E. P. Giannelis, Adv. Funct. Mater. 15, 1285 (2005).

${ }^{13}$ A. B. Bourlinos, E. P. Giannelis, Q. Zhang, L. A. Archer, G. Floudas, and G. Fytas, Eur. Phys. J. E 20, 109 (2006). 
${ }^{14}$ R. Rodriguez, R. Herrera, L. A. Archer, and E. P. Giannelis, Adv. Mater. 20, 4353 (2008).

${ }^{15}$ P. Agarwal, H. Qi, and L. A. Archer, Nano Lett. 10, 111 (2010).

${ }^{16}$ H.-Y. Yu and D. L. Koch, Langmuir 26, 16801 (2010).

${ }^{17}$ A. Chremos, A. Z. Panagiotopoulos, H.-Y. Yu, and D. L. Koch, J. Chem. Phys. 135, 114901 (2011).

${ }^{18}$ S. Goyal and F. A. Escobedo, J. Chem. Phys. 135, 184902 (2011).

${ }^{19}$ A. Chremos and A. Z. Panagiotopoulos, Phys. Rev. Lett. 107, 105503 (2011).

${ }^{20}$ E. R. Chan, L. C. Ho, and S. C. Glotzer, J. Chem. Phys. 125, 064905 (2006).

${ }^{21}$ C. R. Iacovella, A. S. Keys, M. A. Horsch, and S. C. Glotzer, Phys. Rev. E 75, 040801 (2007)

${ }^{22}$ Z. Zhang, M. A. Horsch, M. H. Lamm, and S. C. Glotzer, Nano Lett. 3, 1341 (2003).

${ }^{23}$ X. Zhang, Z. L. Zhang, and S. C. Glotzer, Nanotechnology 18, 115706 (2007).

${ }^{24}$ M. A. Horsch, Z. Zhang, and S. C. Glotzer, Nano Lett. 6, 2406 (2006).

${ }^{25}$ M. R. Wilson, A. B. Thomas, M. Dennison, and A. J. Masters, Soft Matter 5, 363 (2009).

${ }^{26}$ A. Jayaraman and K. S. Schweizer, J. Chem. Phys. 128, 164904 (2008).

${ }^{27}$ A. Jayaraman and K. S. Schweizer, Langmuir 24, 11119 (2008).

${ }^{28}$ A. Jayaraman and K. S. Schweizer, Macromolecules 42, 8423 (2009).

${ }^{29}$ P. Akcora, H. Liu, S. K. Kumar, J. Moll, Y. Li, B. C. Benicewicz, L. S. Schadler, D. Acehan, A. Z. Panagiotopoulos, V. Pryamitsyn, V. Ganesan, J. Ilavsky, P. Thiyagarajan, R. H. Colby, and J. F. Douglas, Nature Mater. 8, 354 (2009)

${ }^{30}$ D. Nykypanchuk, M. M. Maye, D. van der Lelie, and O. Gang, Nature (London) 451, 549 (2008).

${ }^{31}$ J. D. Weeks, D. Chandler, and H. C. Andersen, J. Chem. Phys. 54, 5237 (1971).

${ }^{32}$ J. S. Smith, D. Bedrov, and G. D. Smith, Compos. Sci. Technol. 63, 1599 (2003).

${ }^{33}$ S. J. Plimpton, J. Comput. Phys. 117, 1 (1995).

${ }^{34}$ P. J. in't Veld, S. J. Plimpton, and G. S. Grest, Comput. Phys. Commun. 179, 320 (2008).
${ }^{35}$ A. J. C. Ladd, J. Chem. Phys. 93, 3484 (1990).

${ }^{36}$ H. Hasimoto, J. Fluid Mech. 5, 317 (1959).

${ }^{37}$ A. S. Sangani and A. Acrivos, Int. J. Multiphase Flow 8, 343 (1982).

${ }^{38} D_{\mathrm{G}}$ in Fig. 3 is corrected for periodicity effects using Eq. (5) with a volume fraction based on the total size of the particle defined by Eq. (12).

${ }^{39}$ X. C. Chen and P. F. Green, Langmuir 26, 3659 (2009).

${ }^{40}$ I. Borukhov and L. Leibler, Macromolecules 35, 5171 (2002).

${ }^{41}$ S. L. Elliott and W. B. Russel, J. Rheol. 42, 361 (1998).

${ }^{42}$ W. B. Russel, D. A. Saville, and W. R. Schowalter, Colloidal Dispersions (Cambridge University Press, Cambridge, 1989).

${ }^{43}$ J.-P. Hansen and I. R. McDonald, Theory of Simple Liquids (Academic, Cambridge, 2006).

${ }^{44}$ E. W. Fischer, Physica A 201, 183 (1993).

${ }^{45}$ W. Vanmegen and P. N. Pusey, Phys. Rev. A 43, 5429 (1991).

${ }^{46}$ J. Mattsson, H. M. Wyss, A. Fernandez-Nieves, K. Miyazaki, D. R. R. Z. Hu, and D. A. Weitz, Nature (London) 462, 83 (2009).

${ }^{47}$ U. Genz, B. Daguanno, J. Mewis, and R. Klein, Langmuir 10, 2206 (1994).

${ }^{48}$ I. M. Krieger and T. J. Dougherty, Trans. Soc. Rheol. 3, 137 (1959).

${ }^{49}$ D. A. Jones, B. Learly, and D. V. Boger, J. Colloid Interface Sci. 150, 84 (1991).

${ }^{50}$ A. K. Doolittle, J. Appl. Phys. 22, 1471 (1951).

${ }^{51}$ L. V. Woodcock and C. A. Angell, Phys. Rev. Lett. 47, 1129 (1981).

${ }^{52}$ C. N. Likos, H. Löwen, M. Watzlawek, B. Abbas, O. Jucknischke, J. Allgaier, and D. Richter, Phys. Rev. Lett. 80, 4450 (1998).

${ }^{53}$ B. M. Erwin, M. Cloitre, M. Gauthier, and D. Vlassopoulos, Soft Matter 6, 2825 (2010)

${ }^{54}$ T. Pakula, D. Vlassopoulos, G. Fytas, and J. Roovers, Macromolecules 31, 8931 (1998)

${ }^{55}$ B. Lonetti, M. Camargo, J. Stellbrink, C. N. Likos, E. Zaccarelli, L. Willner, P. Lindner, and D. Richter, Phys. Rev. Lett. 106, 228301 (2011).

${ }^{56} \mathrm{D}$. Truzzolillo, D. Vlassopoulos, and M. Gauthier, Macromolecules 44, 5043 (2011).

${ }^{57}$ D. Vlassopoulos, G. Fytas, T. Pakula, and J. Roovers, J. Phys.: Condens. Matter 13, R855 (2001).

${ }^{58}$ T. C. McLeish, Adv. Phys. 51, 1379 (2002). 